\title{
COINCIDENCE AND FIXED POINTS FOR COMPATIBLE AND RELATIVELY NONEXPANSIVE MAPS
}

GERALD JUNGCK

\author{
Department of Mathematics \\ Bradley University \\ Peoria, IL 61625
}

(Received July 9, 1991 and in revised form January 2, 1992)

\begin{abstract}
The concept of relatively nonexpansive maps is introduced. Fixed point and coincidence results for families of four self maps of metric spaces are obtained. Non-continuous compatible and relatively nonexpansive maps on star-shaped compact subsets of normed linear spaces are highlighted, and two theorems of Dotson are generalized.
\end{abstract}

KEY WORDS AND PHRASES. Relatively nonexpansive and compatible maps, coincidence points.

1991 AMS SUBJECT CLASSIFICATION CODES. 47H10, 54H25.

\section{INTRODUCTION}

In [1] Dotson proved the following theorem.

THEOREM 1.1 (Dotson). If $\mathrm{T}$ is a nonexpansive self-map of a compact star-shaped subset $\mathrm{C}$ of a Banach space (i.e., $\|T x-T y\| \leq\|x-y\|$ for $x, y \in C$ ), then $T$ has a fixed point in C.

Our intent is to generalize this result. We do so by introducing the concept of relatively nonexpansive functions. Our first result is a fixed point theorem for four self maps of a general metric space, which has known results as corollaries, and is used to prove our main result which pertains to star-shaped compact subsets of a linear space. Of interest is the fact that none of the results in the body of this paper require that functions be continuous; this is effected in part by using the concepts of compatibility and surjectivity.

Self maps $f$ and $g$ of a metric space $(X, d)$ are compatible ([2]) iff whenever $\left\{x_{n}\right\}$ is a sequence in $X$ such that $f x_{n}, g x_{n} \rightarrow t$ for some $t \in X$, then $d\left(f g x_{n}, g f x_{n}\right) \rightarrow 0$. An immediate consequence of the definition (Proposition 2.2, [2]) is that if $f$ and $g$ are compatible and $a \in X$ is a coincidence point of $f$ and $g(f a=g a)$, then $f g a=g f a$. (We shall write $f a$ for $f(a)$ when convenient and confusion is not likely.) In fact, Cor. 2.3 in [3] asserts that whenever $f$ and $g$ are continuous and $(X, d)$ is compact, then $f$ and $g$ are compatible iff $f g a=g f a$ whenever $f a=g a$.

We should also note that a subset $\mathrm{C}$ of a linear space $\mathrm{X}$ is star-shaped iff $\exists q \in C$ such that $t x+(1-t) q \in C$ for $t \in[0,1]$ and $x \in C$. In this event, we shall say that $C$ is star-shaped with respect to $q$. Of course, if $C$ is convex, $C$ is star-shaped with respect to any $q \in C$.

2. A PRELIMINARY RESULT.

We need the following definition. 
DEFINITION 2.1. ([2]) Self maps $A$ and $B$ of a metric space $(X, d)$ are $(\epsilon, \delta)-S, T-$ contractions relative to maps $S, T: X \rightarrow X$ iff $A(X) \subseteq T(X), B(X) \subseteq S(X)$, and there is a function $\delta:(0, \infty) \rightarrow(0, \infty)$ such that $\delta(\epsilon)>\epsilon$ for all $\epsilon$, and for all $x, y \in X$ we have:

(i) $\quad \epsilon \leq d(S x, T y)<\delta(\epsilon)$ implies $d(A x, B y)<\epsilon$, and

(ii) $A x=B y$ whenever $S x=T y$.

THEOREM 2.1. Let $S$ and $T$ be self maps of a metric space $(X, d)$, and let the pair $A, B$ be $(\epsilon, \delta)-S, T$-contractions. If $T(X)$ is complete, there exist $u, v, p \in X$ such that $A u=S u=p=B v=T v$. If furthermore, the pair $A, S(B, T)$ is compatible, then $A p=S p=p(B p=T p=p)$. And if both pairs $A, S$ and $B, T$ are compatible, $p$ is the unique common fixed point of $A, B, S$, and $T$.

PROOF. Since $A$ and $B$ are $(\epsilon, \delta)-S, T$ - contractions, $A(X) \subseteq T(X)$ and $B(X) \subseteq S(X)$, and as a consequence of (i) and (ii) in the definition we know that

$$
S x=T y \text { implies } A x=B y \text {, and } d(A x, B y)<d(S x, T y) \text { if } S x \neq T y .
$$

In particular, $d(A x, B y) \leq d(S x, T y)$ for $x, y \in X$. Let $x_{0} \in X$. For $n \in N$, let $y_{2 n-1}=T x_{2 n-1}=A x_{2 n-2}$ and $y_{2 n}=S x_{2 n}=B x_{2 n-1}$. Since $A(X) \subseteq T(X)$ and $B(X) \subseteq S(X)$, the $x_{i}$ can be so chosen. By Lemma 3.1 in [2], the sequence $\left\{y_{n}\right\}$ thus inductively defined is a Cauchy sequence. But then the sequence $\left\{y_{2 n-1}\right\}$, which is in $T(X)$, is also Cauchy. Since $T(X)$ is complete, $\left\{y_{2 n-1}\right\}$ converges to a point $p=T v$ for some $v \in X$. Therefore, $y_{n} \rightarrow p$. Now (2.1) implies that for $n \in N$ :

$$
d(p, B v) \leq d\left(p, A x_{2 n}\right)+d\left(A x_{2 n}, B v\right) \leq d\left(p, A x_{2 n}\right)+d\left(S x_{2 n}, T v\right) .
$$

Since $\left\{y_{n}\right\}$, and hence any subsequence thereof, converges to $p,(2.2)$ and the definition of $\left\{y_{n}\right\}$ imply that $d(p, B v)=0$; i.e., $p=B v=T v$. But $B(X) \subseteq S(X)$, so there exists $u \in X$ such that $\underline{S u}=B v=\underline{T v}$ (2.1) therefore implies that $A u=B v$. We have:

$$
p=B v=T v=S u=A u .
$$

Thus, the first conclusion of the theorem is verified. If moreover, $A$ and $S$ are compatible, (2.3) implies that $A S u=S A u$, or $A p=S p$. In fact, $p=A p$. Otherwise, $T v \neq S p$, so (2.1) yields $d(p, A p)=d(B v, A p)<d(T v, S p)=d(p, A p)$, a contradiction. We thus have, $p=A p=S p$. Similarly, $p=B p=T p$, provided $B$ and $T$ are compatible. If both pairs $A, S$ and $B, T$ are compatible, the fact that $p$ is the only common fixed point of $A, B, S$, and $T$ follows easily from (2.1).

COROLLARY 2.1. Let $A, B, S$, and $T$ be self maps of a complete metric space $(X, d)$, and suppose that $S$ and $T$ are surjective. If $\exists r \in(0,1)$ such that for $x, y \in X$ :

$$
d(A x, B y) \leq r d(S x, T y),
$$

then $\exists u, v, p \in X$ such that $A u=S u=p=B v=T v$. If moreover, the pairs $A, S$ and $B, T$ are each compatible, then $A, B, S$, and $T$ have a unique common fixed point.

PROOF. Define $\delta:(0, \infty) \rightarrow(0, \infty)$ by $\delta(t)=t / r$.

To highlight the central role of compatibility, we observe

COROLLARY 2.2. Let $A$ and $S$ be compatible self maps of a complete metric space $(X, d)$. If $S$ is surjective and if $\exists r \in(0,1)$ such that $d(A x, A y) \leq r d(S x, S y)$ for $x, y \in X$, then $A$ and $S$ have a unique common fixed point.

The major conclusion of Theorem 1 by Park [4] follows from Theorem 2.1 with $A=B$ and $S=T$. Since the identity map $i(x)=x$ commutes with and is therefore compatible with any map $f: X \rightarrow X$, Theorem 1 of Rhoades [5] is a consequence of Corollary 2.1 with $A=B=i$. 


\section{RELATIVELY NONEXPANSIVE MAPS.}

Sehie Park [6] defined a self map $g$ of a metric space $(X, d)$ to be $f$-nonexpansive if $\exists$ a continuous self map $f$ of $(X, d)$ such that $d(g x, g y) \leq d(f x, f y)$ for $x, y \in X$. Since we wish to extend the concept to four functions and drop the continuity requirement we shall say:

DEFINITION 3.1. Let $A, B, S$, and $T$ be self maps of a metric space $(X, d)$. $A$ and $B$ are nonexpansive (n.e.) relative to $S$ and $T$ iff $d(A x, B y) \leq d(S x, T y)$ for $x, y \in X$. Of course, if $A=B$ and $S=T$, we shall say that $A$ is nonexpansive relative to $S$.

Note that "order" is crucial in this definition. Thus, the pair $A, B$ may be nonexpansive relative to the pair $S, T$, whereas the pair $B, A$ may not be n.e. relative to the pair $S, T$ (See [2], Remark 3.1).

We now state and prove our main result.

THEOREM 3.1. Let $A, B, S, T$ be self maps of a compact subset $C$ of a normed linear space $X$, and suppose that $C$ is star-shaped with respect to $q \in C$. If $S$ and $T$ are surjective and if for all $x, y \in C$ :

$$
\|A x-B y\| \leq\|S x-T y\|,
$$

then there exist $p, t, z \in C$ such that $B z=T z=p=A t=S t$. If $A$ and $S(B$ and $T$ ) are compatible, then $A p=S p(B p=T p)$.

PROOF. Let $k_{n} \in(0,1)$ such that $k_{n} \rightarrow 1$, and for $n \in N$ and $x \in C$ define:

$$
A_{n} x=k_{n} A x+\left(1-k_{n}\right) q, \text { and } B_{n} x=k_{n} B x+\left(1-k_{n}\right) q .
$$

Since $A, B: C \rightarrow C$ and $C$ is star-shaped with respect to $q,(3.2)$ assures us that $A_{n}, B_{n}: C \rightarrow C$. Moreover, (3.2) and (3.1) imply that for all $x, y \in C$ and fixed $n \in N:\left\|A_{n} x-B_{n} y\right\|$ $=k_{n}\|A x-B y\| \leq k_{n}\|S x-T y\|$. But $C=S(C)=T(C)$ is compact and therefore complete, and $0<k_{n}<1$; consequently, Corollary 2.1 implies that for each $n \in N$ there exist $x_{n}, y_{n} \in C$ such that:

$$
A_{n} x_{n}=S x_{n}=p_{n}=B_{n} y_{n}=T y_{n} .
$$

Since $C$ is compact, there is a subsequence $\left\{i_{n}\right\}$ such that $A_{i_{n}} x_{i_{n}}=S x_{i_{n}} \rightarrow p \in C$. However, $\left\|A x_{i_{n}}-S x_{i_{n}}\right\|=\left\|A x_{i_{n}}-A_{i_{n}} x_{i_{n}}\right\|=\left\|A x_{i_{n}}-\left(k_{i_{n}} A x_{i_{n}}+\left(1-k_{i_{n}}\right) q\right)\right\| \leq\left(1-k_{i_{n}}\right)\left\|A x_{i_{n}}\right\|+\left(1-k_{i_{n}}\right)\|q\|$ $\leq\left(1-k_{i_{n}}\right) M$ for some $M>0$ by (3.2) and (3.3) since $C$ is bounded. Therefore, $\left\|A x_{i_{n}}-S x_{i_{n}}\right\| \rightarrow 0$ as $k_{i_{n}} \rightarrow 1$, so that

$$
A x_{i_{n}}, S x_{i_{n}} \rightarrow p \text { as } n \rightarrow \infty \text {. }
$$

Now since $C=T(C), T z=p$ for some $z \in C$. By (3.1) we also have

$$
\|B z-p\| \leq\left\|B z-A x_{i_{n}}\right\|+\left\|A x_{i_{n}}-p\right\| \leq\left\|T z-S x_{i_{n}}\right\|+\left\|A x_{i_{n}}-p\right\| .
$$

Since $T z=p,(3.4)$ and the preceding inequalities imply that $p=B z=T z$. By a similar argument it follows that $A t=S t=p$ for some $t \in C$.

If moreover, $A$ and $S$ are compatible, $A t=S t=p$ implies that $S A t=A S t$; i.e., $S p=A p$. In like fashion, if $B$ and $T$ are compatible, $T_{p}=B p$. /

If $A=B$ in the statement of Theorem 3.1 and $A$ is also injective, then $B z=A t$ implies that $t=z$, and we have $A z=S z=T z$. So we can say the following. 
COROLLARY 3.1. Let $A, S$, and $T$ be self maps of a compact subset $C$ of a normed linear space $X$, and suppose that $C$ is star-shaped with respect to $q \in C$. If $S$ and $T$ are surjective and

$$
\|A x-A y\| \leq\|S x-T y\| \text { for } x, y \in C,
$$

then $\exists p \in C$ such that $A p=S p=T p$ provided one of (a) and (b) below obtains:

(a) $A$ is one-to-one,

(b) $A, S$ and $A, T$ are compatible pairs.

With $S=T$ in Corollary 3.1, we obtain:

COROLLARY 3.2. Let $A$ and $S$ be compatible self maps of a compact star-shaped subset $C$ of a normed linear space. If $S$ is surjective and $A$ is nonexpansive relative to $S$, then $A$ and $S$ have a coincidence point.

Again, with $A=i$, the identity map, we have by Corollary 3.1 :

COROLLARY 3.3. If $S$ and $T$ are surjective self maps of a star-shaped compact subset $C$ of a normed linear space $X$ such that $\|x-y\| \leq\|S x-T y\|$ for $x, y \in C$, then $p=S p=T p$ for some $p \in C$.

Of course, the fixed point $p$ of Corollary 3.3 need not be unique; e.g., let $S=T=i$.

Note that Dotson's result, Theorem 1.1, which states that any nonexpansive self map of a starshaped compact subset of a normed linear space has a fixed point, follows from Corollary 3.1(b) with $S=T=i$, the identity map.

Our next theorem extends Dotson's Theorem 2. [1] for weakly compact sets. We shall use the symbol $\stackrel{w}{\rightarrow}$ to denote weak convergence. We sketch the proof since it is similar to that of Theorem 3.1 and it incorporates ideas used in the proof of Dotson's Theorem 2. We remind the reader that a mapping $S: C \rightarrow X$ of a subset $C$ of a Banach space $X$ is demi-closed provided whenever $\left\{x_{n}\right\} \subseteq C$, $x_{n} \stackrel{w}{\rightarrow} \in C$, and $S x_{n} \rightarrow y \in X$, then $S x=y$.

THEOREM 3.2. Let $A, B, S$, and $T$ be self maps of a weakly compact subset $C$ of a Banach space $X$, and suppose that $C$ is star-shaped with respect to $q \in C$. If $S$ and $T$ are surjective and the pair $A, B$ is nonexpansive relative to the pair $S, T$, there exists $x(y) \in C$ such that $A x=S x(B y=T y)$ provided $S-A(T-B)$ is demi-closed.

PROOF. Define $k_{n}, A_{n}$, and $B_{n}$ as in the proof of Theorem 3.1. Since $C=T(C)$ is weakly compact and therefore norm-closed, $C$ is complete. Consequently, the argument given in the proof of Theorem 3.1 up to (3.3) pertains and $\exists x_{n}, y_{n} \in C$ such that (3.3) holds. Since $C$ is weakly compact and $x_{n}, A_{n} x_{n} \in C$ for $n \in N$, there exists a subsequence $\left\{i_{n}\right\}$ such that $x_{i_{n}} \underset{w}{x}$ and $A_{i_{n}} x_{i_{n}}=S x_{i_{n}} \stackrel{w}{\rightarrow} p$ for some $x, p \in C$. And as in the proof of Theorem 3.1, we' have for all $n$ :

$$
\left\|A x_{i_{n}}-S x_{i_{n}}\right\| \leq\left(1-k_{i_{n}}\right)\left\|A x_{i_{n}}\right\|+\left(1-k_{i_{n}}\right)\|q\| .
$$

Since weakly convergent sequences are norm-bounded and since $k_{i_{n}} \rightarrow 1$, (3.6) implies that $(A-S) x_{i_{n}} \rightarrow 0$. But $x_{i_{n}} \stackrel{w}{x}$ and $A-S$ is demi-closed, so that $(A-S) x=0$; i.e., $A x=S x$. Similarly, $T y=B y$ for some $y \in C$ if $B-T$ is demi-closed, so that $(A-S) x=0$; i.e., $A x=S x$. Similarly, $T y=B y$ for some $y \in C$ if $B-T$ is demi-closed. /

COROLLARY 3.4. Let $A$ and $S$ be self maps of a weakly compact subset $C$ of a Banach space $X$ and suppose that $C$ is star-shaped with respect to $q \in C$. If $S$ is surjective, $A$ is nonexpansive relative to $S$, and if $A-S$ is demi-closed, then $A p=S p$ for some $p \in C$.

4. RETROSPECT.

It is known-as a consequence of Theorem 3.2 in [3]-that if $f$ and $g$ are continuous and compatible self maps of a compact metric space $(X, d)$ such that $f(X) \subseteq g(X)$ and $d(f x, f y)<d(g x, g y)$ 
when $g x \neq g y$, then $\exists$ a unique $x \in X$ such that $f x=g x=x$. In view of Corollary 3.2 above, we are prompted to ask under what circumstances we could relax the preceding strict inequality by merely requiring that $f$ be nonexpansive relative to $g$, and still obtain a common fixed point for $f$ and $g$. The next theorem provides a possible beginning point for such inquiry. We prove this theorem by appeal to the following lemma, which lemma is actual proved in [7].

LEMMA 4.1 ([8]) Let $f, g: I \rightarrow I$ be continuous and compatible. If $f$ and $g$ have a coincidence point but no common fixed points, there exists $a, b \in I=[0,1]$ such that

(i) $f(a)=g(a) \geq b<a \geq f(b)=g(b)$, and

(ii) $f(x)<g(x)$ for $x \in(a, b)$.

THEOREM 4.1. Let $f$ and $g$ be continuous, compatible self maps of the interval $I=[0,1]$ such that $f(I) \subseteq g(I)$. If $f$ is nonexpansive relative to $g$, then $f x=g x=x$ for some $x \in I$.

PROOF. Since $f$ and $g$ are continuous and $f(I) \subseteq g(I), f x=g x$ for some $x \in I$. Suppose that $f$ and $g$ have no common fixed point. Then $\exists a, b \in I$ such that (i) and (ii) of Lemma 4.1 hold. Since $g(a)>a$ and $g(b)<b, \quad \exists c \in(a, b)$ such that $g(c)=c$. But then (i) and (ii) imply that $f(c)<g(c)=c<b \leq f(a)=g(a)$; i.e., $|f(a)-f(c)|>|g(a)-g(c)|$, a contradiction. /

The following examples demonstrate that each of the hypothesized properties in the above theorem fills a necessary role (In none of the examples cited does the defined pair, $f, g$ have a common fixed point.). In each example, $I=[0,1]$.

EXAMPLE 4.1. Let $f(x)=1$ and $g(x)=1-x$ for $x \in I$. Then $f$ and $g$ satisfy all aspects of the hypothesis of Theorem 4.1 except compatibility. Note that $f(0)=g(0)=1$, but $f g(0)=f(1)$ $=1 \neq 0=g(1)=g f(0)$.

EXAMPLE 4.2. Let $g(x)=(2 x$ on $[0,1 / 2], 1$ on $[1 / 2,1]$, and $1 / 2$ at $x=1)$ and let $f(x)=(1-2 x$ on $[0,1 / 4]$ and $g(x)$ on $[1 / 4,1])$. We assert that $f$ and $g$ satisfy the hypothesis of Theorem 4.1 except for "continuity". Since $f=g$ and $f g=g f$ on $[1 / 4,1]$, the only occasion for concern regarding compatibility would be $f x_{n}, g x_{n} \rightarrow 1 / 2$, in which instance $g f x_{n}, f g x_{n} \rightarrow 1$, thereby confirming compatibility.

EXAMPLE 4.3. Let $f(x)=1-x$ and $g(x)=(1-x)^{2}$ for $x \in I$. Note that $f$ is not n.e. relative to $g$, and conversely. Consider successively: $x=1 / 2, y=1$, and $x=0, y=1 / 2$. On the other hand, the remainder of the hypothesis is satisfied (e.g., $f$ and $g$ commute at $x=0,1$, their point of "coincidence" ).

QUESTION. What compacta can be substituted for $I$ in Theorem 4.1 and still yield a true result?

\section{REFERENCES}

1. DOTSON, JR, W.G., Fixed point theorems for non-expansive mappings on star-shaped subsets of Banach spaces, J. London Math. Soc. (2) 4 (1972), 408-410.

2. JUNGCK, G., Compatible mappings and common fixed points, Internat. J. Math. Math. Sci. 9 (1986), 771-779.

3. - - Common fixed points for commuting and compatible maps on compacta, Proc. Amer. Math. Soc. 103 (1988), 977-983.

4. PARK, S., A coincidence theorem, Bull. de L'Academie Polonaise Des Sciences 29 (1981), 487-489.

5. RHOADES, B.E., Some fixed point theorems for pairs of mappings, Jnanabha 15 (1985), 151156. 
6. PARK, S., On $f$-nonexpansive maps, J. Korean Math. Soc. 16 (1979), 29-38.

7. JUNGCK, G., Commuting mappings and common fixed points, Amer. Math. Monthly 73 (1966), 735-738.

8. Soc. 115 (1992), 495-499. 


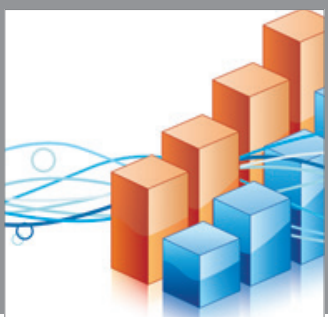

Advances in

Operations Research

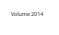

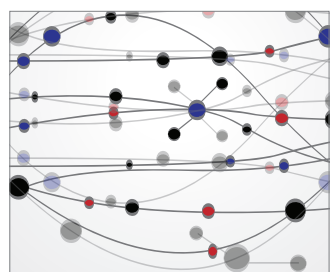

\section{The Scientific} World Journal
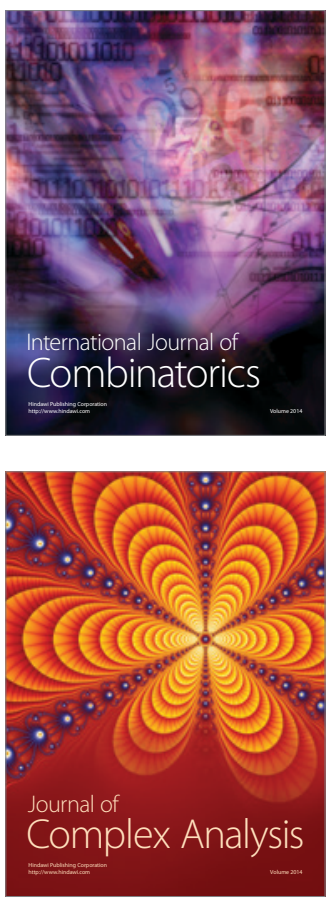

International Journal of

Mathematics and

Mathematical

Sciences
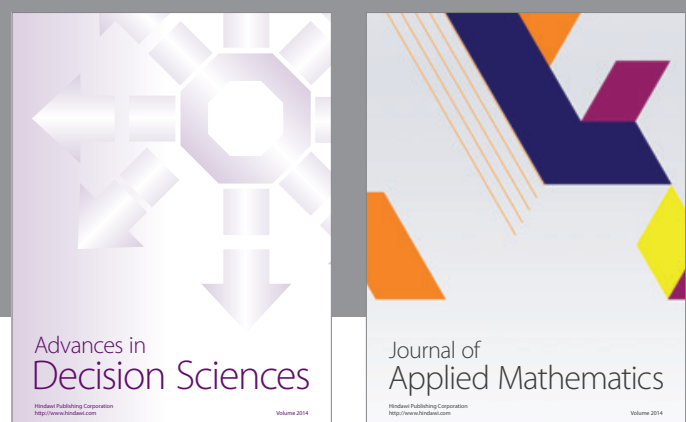

Journal of

Applied Mathematics
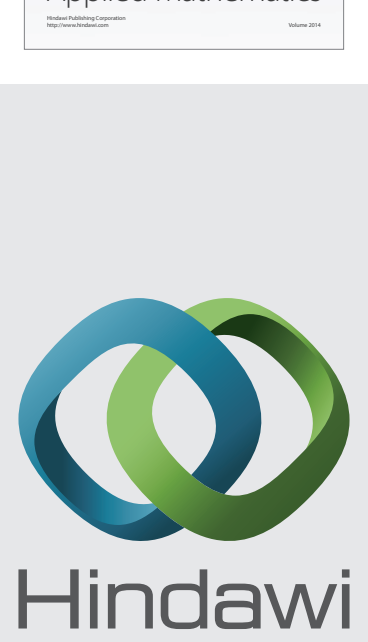

Submit your manuscripts at http://www.hindawi.com
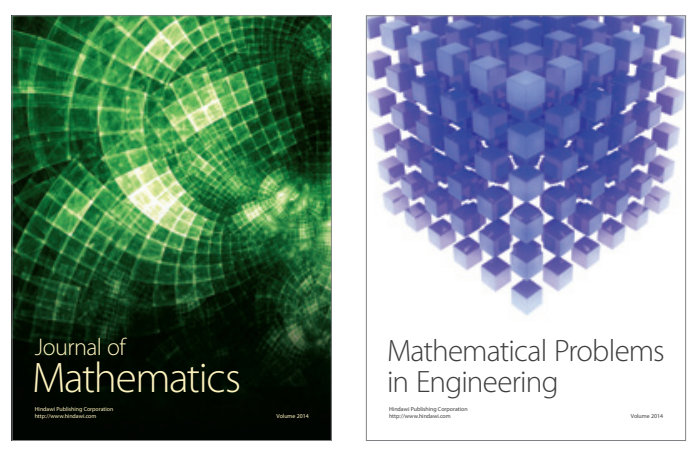

Mathematical Problems in Engineering
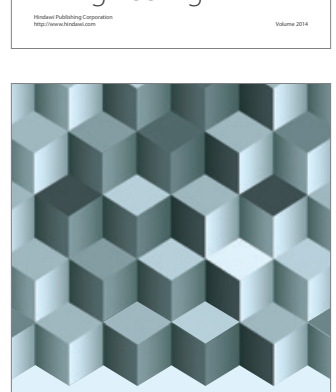

Journal of

Function Spaces
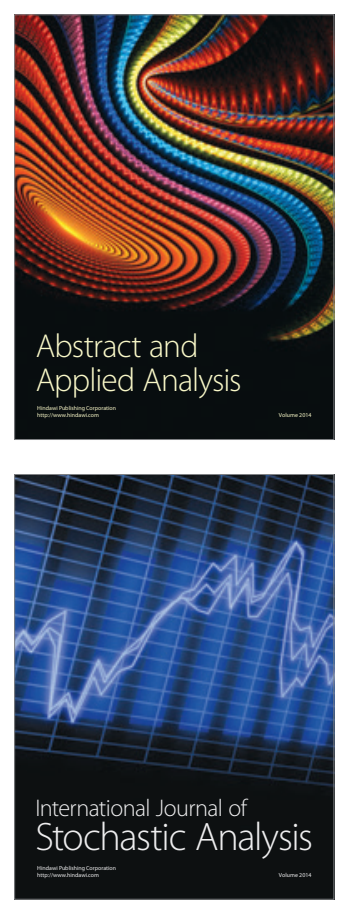

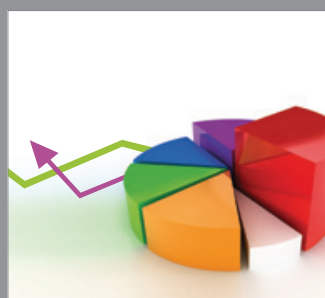

ournal of

Probability and Statistics

Promensencen
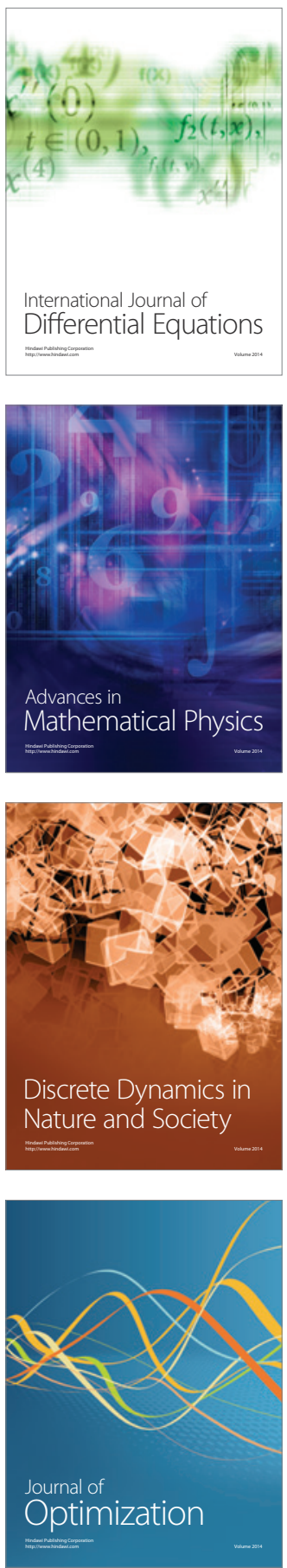